\title{
La Sante des Populations des Espaces Frontaliers au Sud-Est du Benin: Quels Defis Sanitaires dans un Espace Cosmopolite?
}

\author{
Bio Bigou Bani Léon, \\ Professeur Titulaire des Universités (CAMES) \\ Oladjide Azaria, \\ Djinadou O. A. Rachade, \\ Doctorants à l'Ecole Doctorale Pluridisciplinaire « Espace- Culture et \\ Développement » Laboratoire d'Etudes des Dynamiques Urbaines et \\ Régionales (LEDUR), Universite d`Abomey-Calavi
}

Doi: 10.19044/esj.2019.v15n6p391～URL:http://dx.doi.org/10.19044/esj.2019.v15n6p391

\section{Résumé}

La complexité des milieux frontaliers nécessite un système spécifique de soins de santé aux populations résidentes. L'analyse des ressources en santé face au caractère cosmopolite des espaces frontaliers a été faite à partir des éléments du rapport final de la cartographie des interventions et intervenants en santé maternelle néo-natale et infanto-juvénile, les données du Système National d'Information et de Gestion Sanitaires actualisées au niveau des zones sanitaires concernées. Les résultats obtenus montrent une grande difficulté de l'offre de santé dans les espaces frontaliers au sud -est du Bénin. L'insuffisance en ressources humaines est importante de façon générale. La couverture des deux zones sanitaires en sages-femmes et infirmiers est satisfaisante. Cependant, leur répartition dans les centres de santé ne tient pas compte des particularités des espaces frontaliers. Il est noté un gap en matériel roulant pour le déplacement en urgence ( 4 ambulances), pour la supervision et le ravitaillement (12 véhicules) ainsi que pour les stratégies avancées (31 motos). L'intervention au niveau famille et communauté requiert encore beaucoup d'attention au regard du nombre important de relais communautaires qui reste à former et à pourvoir en kits de relais ; les kits SMNI sont quasiment inexistants dans presque toutes les formations sanitaires. Au regard de ces constats peu reluisants, il est nécessaire d'adapter l'offre de soins et l'organisation locale du système de santé pour tenir compte de l'ensemble des acteurs présents localement et des besoins des populations transfrontalières. 
Mots-clés: Bénin, santé, cosmopolite, ressources en santé, Nigeria

\title{
Pubic Health of Frontier Spaces in the South-East Benin: Which of the Medical Challenges is in a Cosmopolitan Space?
}

\author{
Bio Bigou Bani Léon, \\ Professeur Titulaire des Universités (CAMES) \\ Oladjide Azaria, \\ Djinadou O. A. Rachade,
}

Doctorants à l'Ecole Doctorale Pluridisciplinaire « Espace- Culture et Développement » Laboratoire d'Etudes des Dynamiques Urbaines et Régionales (LEDUR), Universite d`Abomey-Calavi

\begin{abstract}
The complexity of the frontier mediums requires a specific system of health care to the resident populations. The analysis of the health resources face to the cosmopolitan character of frontier spaces was made starting from the elements of the final report of the cartography of the interventions and speakers in neo-nativity and youthful maternal health, the data of the National System of Medical Information and Management brought up to date on the level of the medical concerned zones. The obtained results show a great difficulty of offer a health care in frontier spaces in the south-east of Benin. The insufficiency in human resources is generally significant. The cover of the two medical zones as midwives and male nurses is satisfactory. However, their distribution in health centers doesn't take into account the frontier spaces characteristics. It is noted a gap in transport material for transportation of urgency case (4 ambulances), for the supervision and the supply (12 vehicles) like for the advanced strategies (31 motor bikes). The intervention on the family and community level still requires much attention in comparison with the significant number of Community relays which remain to be formed and to provide in kits with stage; kits SMNI are almost nonexistent in almost all the medical formations. Taking into consideration these report little glittering, it is necessary to adapt the offer of care and the local organization of the system of health to locally take into account the whole present actors and the transborder populations needs.
\end{abstract}


Keywords: Staff, Radiological facility, Health structures, Central Africa Republic

\section{Introduction}

Situées à la croisée d'enjeux idéologiques, politiques et territoriaux, les frontières et les espaces qu'elles déterminent ont une signification qui dépasse largement leur emprise territoriale limitée (Denert \& Hurel, 2000). La croissance démographique dans ces espaces frontaliers due non seulement au fort taux de naissance, mais aussi et surtout au stock de migrants doit être accompagnée de mesures. Ces mesures ont traits à la scolarisation, à la sécurité, à l'hébergement ... et fondamentalement à la santé des populations. En effet, la mondialisation croissante des échanges engendre une augmentation des flux internationaux de populations humaines, animales et de marchandises qui peuvent favoriser la propagation des maladies et d'autres événements de santé qu'ils soient d'origine biologique, chimique, radiologique ou nucléaire. Selon l'Agence Béninoise de Gestion Intégrée des Espaces Frontaliers (ABeGIEF), «l'enclavement des localités prend une tournure tragique dans le domaine sanitaire. Les structures de santé, quand elles existent, manquent de moyens humains et matériels. Faute de ces moyens, les centres de santé communiquent moins bien que leurs patients. Une épidémie tourne vite à la catastrophe sanitaire faute de moyens de communication entre les centres de santé ». La connaissance intime des situations de santé d'une collectivité ou d'une communauté est un préalable indispensable à la définition d'une politique d'action, aux choix des programmes, à l'adéquation des moyens disponibles aux besoins de santé exprimés ou observés (Benyoussef \& al, 1973).

Les localités transfrontalières du Bénin et de ses voisins sont des régions caractérisées par une forte densité et une grande interconnexion transfrontalière de leurs populations.

Cependant la coopération entre les systèmes de santé des pays en particulier dans le domaine de la surveillance intégrée des maladies et la riposte, n'est pas encore à la hauteur de cette importante interconnexion transfrontalière des populations. La dynamique de la population issue de la fonction des espaces frontaliers augmente la vulnérabilité des individus sur le plan sanitaire. Comment faire face au besoin en matière de santé d'une population entière et en réduire les inégalités entre différents groupes démographiques dans un espace cosmopolite ?

Une communauté transfrontalière se caractérise par une histoire et une culture partagées, ainsi que par la volonté de renforcer le sentiment d'appartenance par des actions concrètes, globales et durables. La santé peut constituer l'un des piliers de cette communauté transfrontalière. Ceci suppose 
l'adaptation de l'offre de soins et l'organisation locale du système de santé qui intéresse l'ensemble des acteurs présents localement, et les besoins de toute la population du bassin de vie transfrontalière. La dernière épidémie de la maladie hémorragique à virus Ebola qu'ont connue quelques pays de l'Afrique de l'ouest en 2016 et celle à virus Lassa apparue aux Nigéria et certaines localités frontalières du Bénin en 2015-2017 ont montré, une fois de plus, la nécessité de renforcer le système sanitaire dans les espaces frontaliers.

Ce chapitre analyse la capacité des zones sanitaires (auxquelles appartiennent les espaces frontaliers des communes d'Adjarra et de SèmèPodji) à répondre aux besoins en santé des populations face à la diversité linguistique et culturelle de ces milieux, à la promiscuité et à la nature des activités qui s'y exercent.

\section{Caractéristiques sociodémographique et ethnographique de la population}

\subsection{Dynamique de l'évolution de la population au sud-est du Bénin}

Les espaces frontaliers au sud-est du Bénin sont constitués par l'ensemble des localités des communes de Sèmè-Podji et d'Adjarra partageant la frontière avec le Nigéria. Au Recensement Général de la population et de l'Habitat $\left(\mathrm{RGPH}_{1}\right)$ de 1979, la zone d'étude comptait 11219 habitants contre 22070 habitants en 1992, soit pratiquement le double en l'espace de 12 ans. En 2002, les résultats du troisième Recensement Général de la Population et de l'Habitat $\left(\mathrm{RGPH}_{3}\right)$ portent l'effectif des populations de la zone à 30363 habitants dont 14278 hommes et 16085 femmes. A l'issue du dernier Recensement Général de la population et de l'Habitat de $2013\left(\mathrm{RGPH}_{4}\right)$, cet effectif est porté à 49116 habitants dont 25143 femmes et 23973 hommes (figure 1).

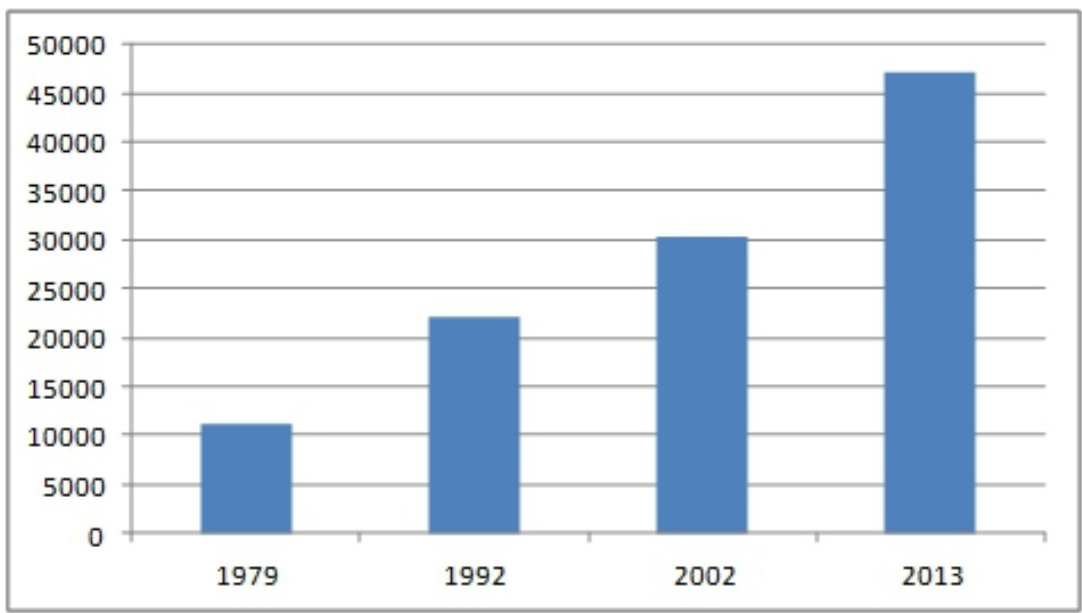

Figure 1: Evolution de la population de 1979 à 2013

Source : INSAE, (Cahiers de villages et quartiers de villes, 1979, 1990, 2002 et 2013) 
La figure 1 présente l'évolution de la population de 1979 à 2013 pour les espaces frontaliers considérés. D'une manière générale, sur l'ensemble de la période, la croissance de la population des espaces frontaliers des communes d'Adjarra et de Sèmè-Podji a été très drastique. Cependant, cette augmentation accélérée de la population a été plus importante dans les espaces arrimées aux frontières de la commune de Sèmè-Podji que ceux d'Adjarra. En trois décennies, la population de ces espaces s'est accrue de $77 \%$. Même si cette croissance démographique n'est pas phénomène récent, elle est davantage marquée ces quinze dernières années. L'augmentation rapide de la population, surtout entre 2002 et -2013 témoigne non seulement d'un fort accroissement naturel, mais aussi et surtout de la forte attraction qu'exerce la zone sur les migrants de Nigéria, du Togo, du Niger, etc.

A l'instar de la population, les ménages ont connu également une évolution aussi bien en nombre qu'en taille au cours de la même période. En effet, le nombre total de ménages de la zone s'est élevé de 2445 ménages en 1979 à 10949 en 2013, en passant par 4865 en 1992 et 6649 en 2002 (figure 2).

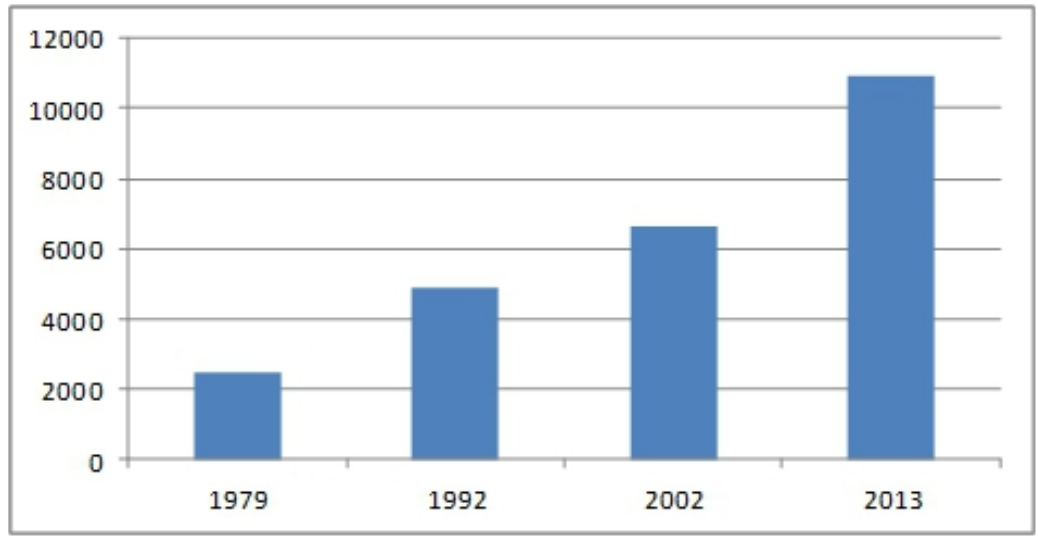

Figure 2: Evolution des ménages de 1979 à 2013

Source : INSAE, (cahiers de villages et quartiers de villes, 1979, 1992, 2002 et 2013)

La figure 2 ci-dessus arbore l'évolution des ménages des espaces frontaliers des communes d'Adjarra et de Sèmè-Podji au sud-est du Bénin. Les ménages ont connu une évolution rapide ces dernières années, surtout entre 2002 et 2013. La fécondité est assurément le facteur qui concourt le plus à l'agrandissement des familles. Cependant, les mouvements migratoires avec leurs flux ont joué un grand rôle dans l'accroissance démographique des espaces au sud-est du Bénin. En effet, toutes les formes de migrations individuelles, (migrations de travail, matrimoniales, enfants confiés, etc.) affectent sensiblement la taille et la structure des ménages. Ainsi des départs au sein d'un ménage sont comblés par des arrivées au sein d'un autre ou la création d'un nouveau ménage. 
Les implications démographiques, sociales, économiques et sanitaires varient selon le type de migration (temporaire, de passage ou définitive), les caractéristiques des migrants, la nature des relations entretenues et l'endroit considéré (ménage de départ ou d'accueil). Le nombre de ménages évolue proportionnellement à l'accroissement de la population (figure 3).

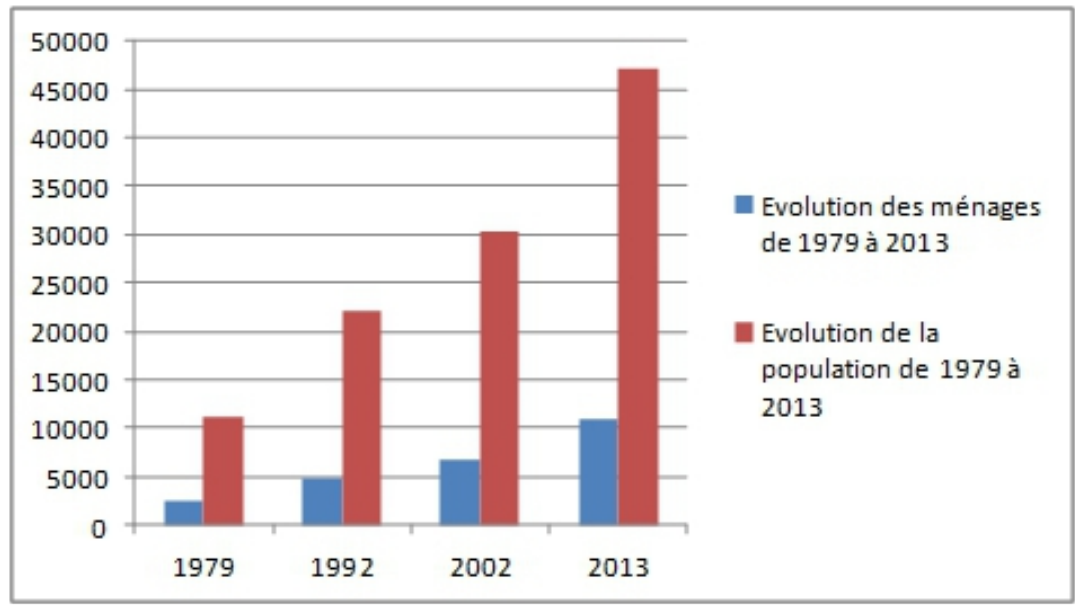

Figure 3: Evolution comparée de la population et du nombre de ménages de 1979 à 2013 Source : INSAE, (cahiers de villages et quartiers de villes, 1979, 1990, 2002 et 2013)

La figure 3 montre une comparaison de l'évolution des populations et des ménages des localités des communes de Sèmè-Podji et d'Adjarra frontalières au Nigéria. En 1979, on dénombrait 2445 ménages pour un effectif de population de 11219 personnes, soit une moyenne de 4,58 personnes par ménage. Il en est de même en 1992. Entre 2002 et 2013, le nombre moyen de personnes par ménage a connu une augmentation non négligeable et est passé à une moyenne de 6 personnes par ménage, avec d'importantes disparités au sein de ces ménages.

\subsection{Distribution des étrangers présents dans les ménages suivant leur origine}

Le sud-est du Bénin est un exemple d'espace où des individus de nationalités différentes, appartenant à des groupes socioculturels différents se côtoient au quotidien. La frontière à Sèmè-Kraké est un carrefour important du couloir commercial reliant Abidjan à Lagos et se caractérise par un flux significatif de personnes et de biens. On y rencontre de multiples nationalités dont les plus représentées (hormis les Béninois considérés comme autochtones) sont les Nigérians, les Togolais, les Nigériens et les Ghanéens (Figure 4). 


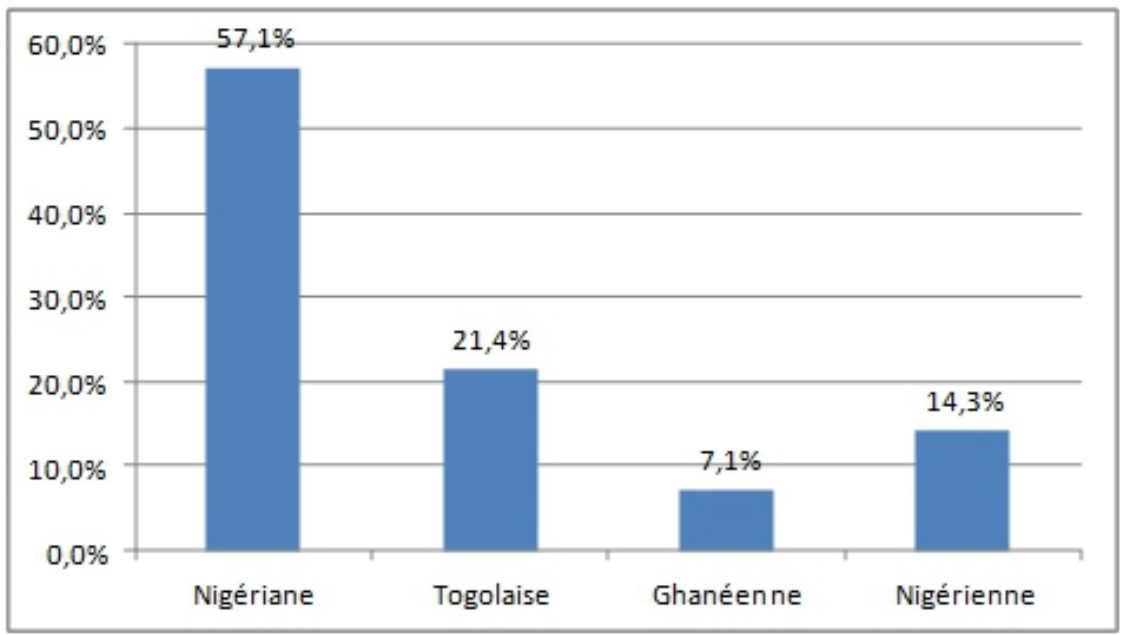

Figure 4: Répartition des étrangers dans les ménages suivant leur origine Source : Enquête de terrain, 2017

La figure 4 ci-dessus expose les grands groupes présents dans les localités des espaces frontaliers des communes de Sèmè-Podji et d'Adjarra. Les Nigerians représentent les groupes les plus importants avec $57 \%$ de l'ensemble des nationalités présentes dans le milieu. Ils sont suivis respectueusement des Togolais avec environ $21 \%$ et les Nigériens (14,3\%). Les Ghanéens sont faiblement présents dans le milieu, mais sont plus importants numériquement que les Ivoiriens et les Burkinabè.

Le redéploiement des espaces frontaliers d'Adjarra et de Sèmè-Podji répond à des mouvements analogues. Toutefois, ceux de Sèmè-Podji ont connu un grand essor grâce à l'aménagement de la route Sèmè-frontière et la réalité du projet Corridor Abidjan-Lagos depuis 2002. Les flux de migration régionale vers le Bénin ont augmenté, selon le profil migratoire national (2011). Les données du recensement de la population et des ménages de 2002 montrent que le nombre d'immigrants est passé de 78000 en 1992 à 157000 en 2002, soit une hausse de plus de $100 \%$ en 10 ans. Le stock d'immigrants en 2002 représentait environ $2 \%$ de la population totale du pays et $5 \%$ de sa population active (INSAE, 2002). Cette importante augmentation du stock d'immigrants entre deux recensements se justifie entre autre par une stabilité politique retrouvée, et la relance des activités économiques à compter de la Conférence des forces vives de la nation de février 1990. Au dernier recensement général de la population et de l'habitation de 2013, le stock de migrants au Bénin est estimé à 234241 personnes dont 131051 hommes et 103190 femmes (figure $5)$. 


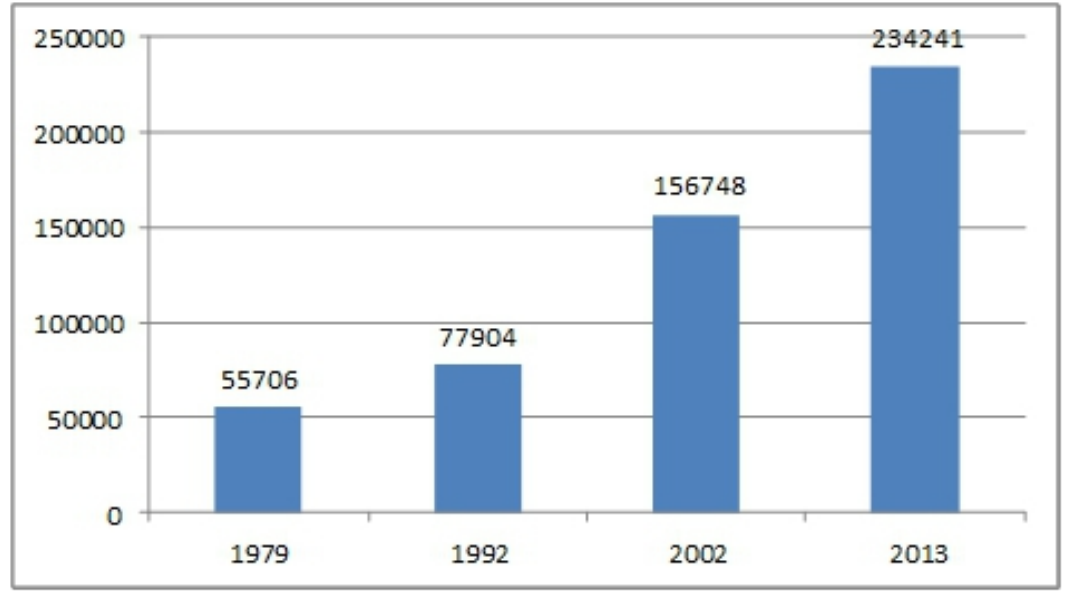

Figure 5: Evolution du nombre d'immigrants au Bénin de 1979 à 2013

Source : INSAE, (cahiers de villages et quartiers de villes, 1979, 1990, 2002 et 2013)

La figure 5 présente l'évolution du nombre d'immigrants au Bénin de 1979 à 2013. En 2013, le nombre d'immigrants fait le triple de celui 1992. L'accroissement important des immigrants entre 1992 et 2002 serait dû à l'instabilité politique de quelques pays de la sous-région comme le Togo et le Nigeria. Mais l'augmentation drastique des immigrants ces dernières années serait essentiellement liée aux opportunités économiques.

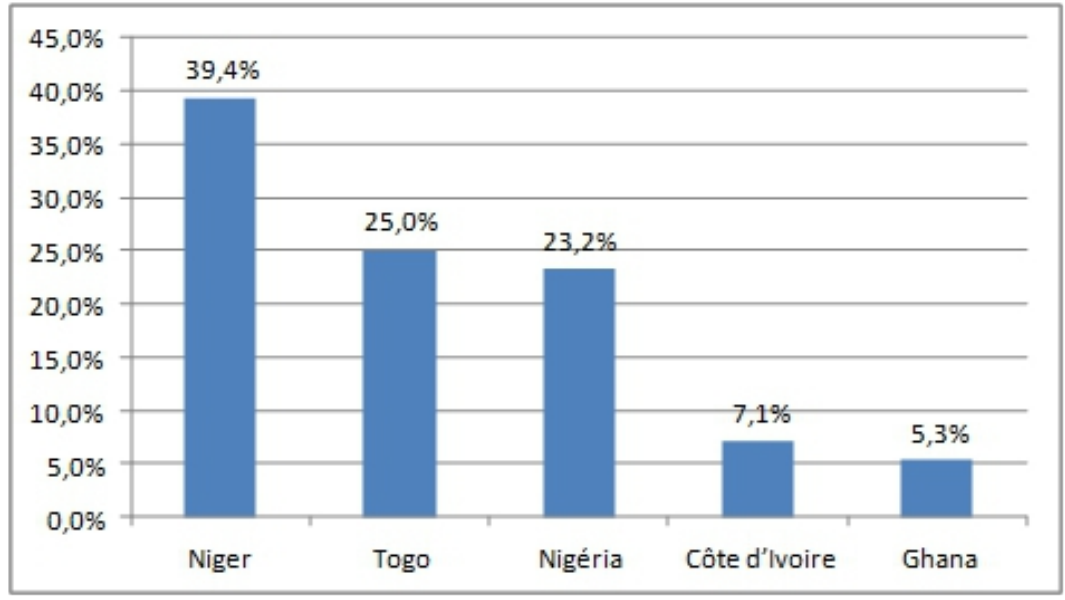

Figure 6: Stock de migrants par origine Source : UNICEF Bénin, migration profiles (2013)

\subsection{Principales langues véhiculaires dans les espaces frontaliers ouest-} Africains

Lorsque dans une même communauté, les populations de différentes nationalités et d'origines ethniques différentes se cohabitent, les comportements en matière d'utilisations des services de soins de santé peuvent 
varier d'un groupe à un autre. La zone d'étude étant un carrefour important, plusieurs groupes sociolinguistiques y séjournent (Figure 7).

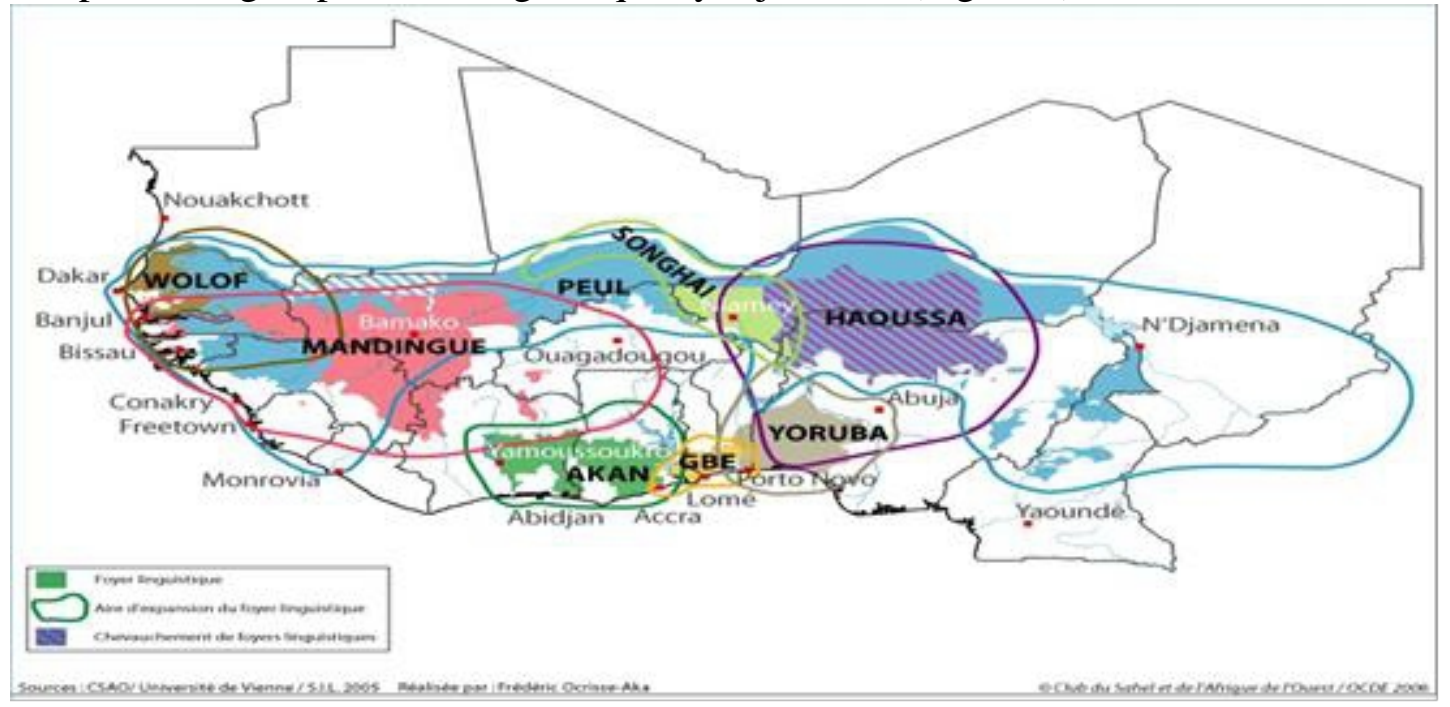

Figure 7: Carte des principales langues véhiculaires de l'Afrique de l'ouest

Source : OCDE, 2011

Les barrières linguistiques constituent d'énormes obstacles dans l'accès aux soins de santé. Dans un espace aussi plurilingue comprenant une population importante de migrants, les interactions entre patients et professionnels de la santé de différentes cultures sont de plus en plus fréquentes. La variable nationalité est donc intervenue pour apprécier les recours aux soins de santé. En effet, il peut exister selon les pays des normes culturelles qui peuvent empêcher les individus (surtout les femmes) de rechercher les soins de santé en dehors de la maison pour elles-mêmes et pour leurs enfants (Rashid \& al, 2001).

Comme la langue, la nationalité, l'attachement aux croyances traditionnelles, aux dogmes, aux interdits et prescriptions, tout comme certaines croyances religieuses influencent parfois le comportement sanitaire (religions animistes/traditionnelles), contribuant ainsi à l'observance d'une négligence de la santé.

\subsection{Distribution des migrants suivant leur durée de vie dans le milieu d'étude}

La migration fait intervenir les notions de zone de départ appelée origine et zone d'arrivée appelée destination. A partir de ces deux notions, la durée de vie a été définie et s'applique à tout individu dont le lieu de résidence à une date de référence donnée est différent de son lieu de naissance. L'analyse des données d'enquête obtenues sur un échantillon de 384 Chefs de ménage 
d'origines étrangères montre que la majorité des personnes étrangères dans le milieu d'étude y résident depuis plus d'une dizaine d'années (figure 8).

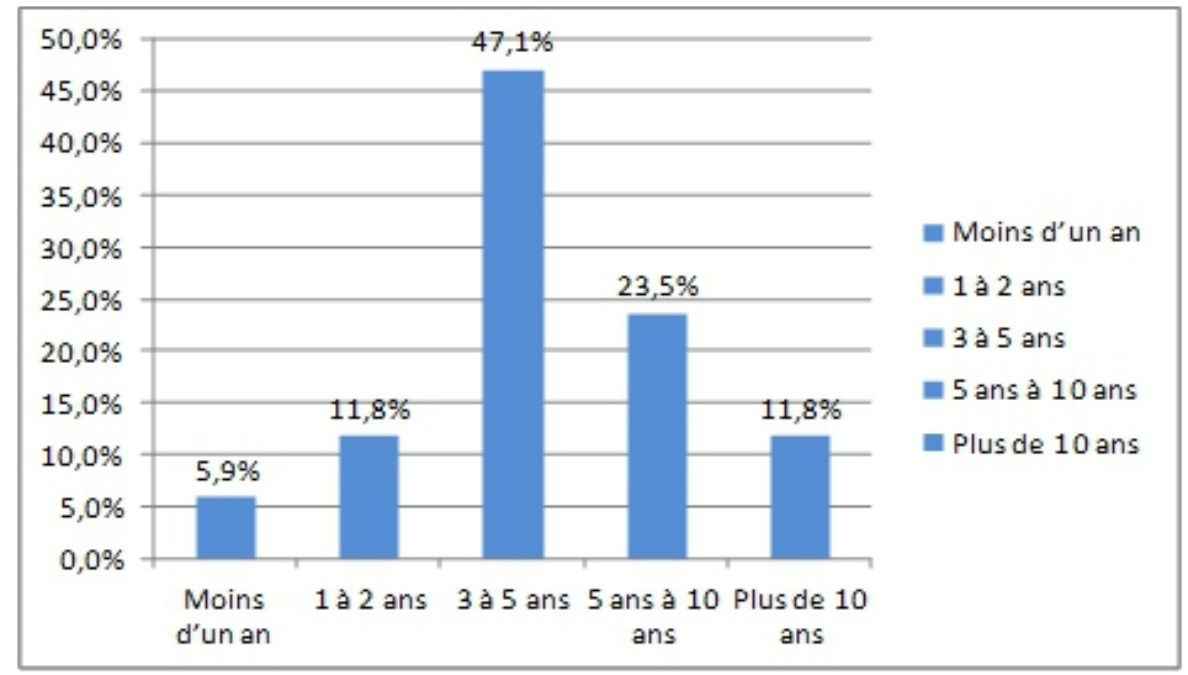

Figure 8: Répartition des étrangers suivant la durée de vie dans le milieu d'étude Source : Enquête de terrain, Juin 2017

La figure 8 présente la répartition des étrangers suivant la durée de vie dans le milieu. Environ $6 \%$ seulement ont une durée de résidence de moins d'un an dans le milieu de résidence. La majorité des personnes étrangères présentes dans le milieu y résident depuis plus de 3 ans. La plupart de ceux qui ont passé plus d'années dans la zone sont des Nigérians.

\section{Système sanitaire face à la vulnérabilité de l'espace frontalier}

Les espaces frontaliers au sud-est du Bénin appartiennent administrativement à deux (2) zones sanitaires à savoir la zone sanitaire PortoNovo-Sèmè-Podji-Aguégués (PAS) et la zone sanitaire Akpro-missérétéAvrankou-Adjarra (AAA). En effet, suite à l'adoption de la stratégie des soins de santé primaires à la Conférence d'Alma-Ata, la réflexion entamée à la Conférence de Lusaka et poursuivie à la Conférence d'Harare a abouti à la recommandation de la création, dans chaque pays, d'entités opérationnelles de mise en œuvre des soins de santé primaires appelés districts sanitaires et disposant d'une large autonomie dans la planification et la gestion des activités du niveau périphérique. Le Bénin étant initialement subdivisé en districts, subdivisions territoriales appelées aujourd'hui commune et, étant entendu que l'aire de santé retenue pour un district peut dépasser les limites d'un ancien district administratif, il fut nécessaire de trouver une autre appellation qui traduit les mêmes réalités politiques, juridiques, sociologiques et économiques que le District Sanitaire. C'est ainsi que l'appellation de «zone sanitaire» a été retenue pour remplacer celle de «district sanitaire» adoptée par l'OMS. 
Les zones sanitaires devaient, autant que possible, respecter les limites des communes, mais pouvaient regrouper si nécessaire plusieurs d'entre elles. $\mathrm{Au}$ plan structurel, la zone sanitaire réunit un ensemble de services périphériques (UVS, CCS, CSSP/CSCU). L'hôpital de zone constitue le niveau de référence de cet ensemble. Au plan de la réorganisation de la base de la pyramide, il a été créé 34 zones sanitaires. Le fonctionnement des zones sanitaires est régi par les principes relatifs à l'objectif de Santé pour tous, définis lors de la Conférence d'Alma Ata :

- la couverture universelle de la population par des soins répondant aux besoins ;

- des services axés sur la promotion, la prévention, le traitement et la réadaptation ;

- des services efficaces, acceptables sur le plan culturel, abordables sur le plan financier et faciles à gérer ;

- la participation de la communauté au développement des services afin d'encourager l'auto responsabilité et diminuer la dépendance ;

- la prise en compte d'autres secteurs de développement dans les approches de la santé.

L'analyse de la situation sanitaire de la zone d'étude est intégrée dans les deux (2) zones sanitaires pour évaluer la capacité du système à réagir dans les espaces frontaliers.

\subsection{Présentation du système de santé}

Le système sanitaire repose sur trois niveaux :

- le niveau périphérique avec la zone sanitaire (familles / communauté ; centre de santé et l'hôpital de zone qui est la $1^{\text {ère }}$ référence);

- le niveau intermédiaire avec la Direction Départementale de la Santé (DDS) et le Centre Hospitalier Départemental ou l'hôpital de $2^{\text {ieme }}$ référence ;

- le niveau central avec les Directions Techniques Centrales (DTC) et la référence nationale ou le Centre National Hospitalier Universitaire (CNHU).

Ces trois niveaux sont liés et s'articulent dans une dynamique qui fonctionne de la périphérie vers le niveau central.

Dans cette logique, les premiers soins se donnent au niveau famille/communauté par les relais communautaires (promotion des pratiques familiales essentielles, soutien aux équipes de santé en stratégie avancée et mobile, prise en charge des affections courantes de l'enfant etc.). 
- Les centres de santé, constituent le premier contact, ils peuvent offrir des services (activités curatives, préventives, promotionnelles et de réadaptation) aux populations environnantes grâce à des stratégies avancées/mobiles.

- L'hôpital de zone (HZ) est la structure de $1^{\text {ère }}$ référence offrant des prestations en matière d'urgences médico-chirurgicales, des Soins Obstétricaux et Néonatals d'Urgence Complet (SONUC), des explorations diagnostiques et hospitalisations.

- Le Centre Hospitalier Départemental (CHD) en tant que structure de $2^{\text {ième }}$ référence, dispose d'un plateau technique plus large pour assurer la prise en charge des cas qui dépassent la compétence de la $1^{\text {ère }}$ référence.

- Le Centre National Hospitalier Universitaire (CNHU) représente la structure de référence nationale. Il assure la prestation de soins de santé hautement spécialisés et sert de centre d'encadrement et de formation pratique des cadres médicaux et paramédicaux.

Le système sanitaire au niveau des espaces frontaliers au sud-est du Bénin ne comporte qu'un seul niveau: le niveau périphérique, mais appartiennent à deux (2) différentes zones sanitaires comportant le niveau intermédiaire. Le niveau périphérique constitue la base de la pyramide sanitaire et compte 34 zones sanitaires réparties sur toute l'étendue du territoire national.

La zone sanitaire représente l'entité opérationnelle la plus décentralisée du système de santé. Elle est organisée sous forme d'un réseau de services publics de premier contact (Maternités et dispensaires seuls, Centres de Santé) et des formations sanitaires privées, le tout appuyé par un hôpital de première référence public ou privé (hôpital de zone) et destiné à desservir une aire qui abrite entre 100.000 et 200.000 habitants.

\subsection{Analyse des ressources en santé disponibles}

L'objectif général poursuivi par le système sanitaire est d'améliorer l'état de santé et le bien-être de la population par une meilleure adéquation qualitative et quantitative entre l'offre des prestations sanitaires et les besoins essentiels de la population. Cette tâche devient encore plus complexe dans un espace cosmopolite où des individus présentent parfois des caractéristiques sanitaires particulières liées à leur provenance. Pour mieux répondre aux priorités sanitaires dans un milieu aussi vulnérable, l'analyse des ressources aussi bien humaines, techniques, matérielles et financiers s'impose. 


\subsubsection{Disponibilité en ressources humaines}

Dans les deux (2) zones sanitaires auxquelles appartient la zone d'étude, le nombre de médecins disponibles est acceptable, mais leur répartition pose problème. En effet, pendant que la zone sanitaire de PortoNovo-Sèmè-Podji-Aguégués (PAS) enregistre un surplus en médecin, on note un gap d'environ 20 médecins dans la zone sanitaire d'Avrankou- ApkroMissérété-Adjarra. Le tableau 1 présente la situation du personnel médical dans les deux zones sanitaires.

Tableau 1: Situation du personnel médical dans les deux zones sanitaires

\section{Zones sanitaires}

\section{Ressources}

humaines

\begin{tabular}{|l|l|}
\hline Médecins \\
\hline Relais communautaires \\
\hline Infirmiers
\end{tabular}

Porto-Novo-Sèmè-PodjiAguégués (PAS)

Gap -33

Gap 784

Gap -36
Avrankou- Ap-kroMissérété-Adjarra Méd : Gap 23

RC : Gap 1490

Infir : Gap 0

Source : Cartographie des intervenants et interventions en SMNI actualisée par les ZS. Juin 2017

L'analyse du tableau 1 montre que la couverture des deux zones sanitaires en sages-femmes et infirmiers est satisfaisante. Cependant, leur répartition dans les centres de santé doit tenir compte des particularités des espaces frontaliers. Par contre, les relais communautaires dont la fonction est d'une importance et d'une nécessité absolue dans la mise en œuvre des plans sanitaires au niveau famille et communauté sont presque absents dans toutes les localités. Le gap est très prononcé et est de l'ordre de 5000 relais communautaires à former et à pourvoir en kits de relais. Le tableau 2 présente les interventions traceurs et les niveaux de prestation des soins.

Tableau 2: Interventions traceurs par niveau de prestation des soins

\begin{tabular}{|l|l|l|}
\hline $\mathbf{N}^{\circ}$ & \multicolumn{1}{|c|}{ Interventions traceurs } & $\begin{array}{l}\text { Niveau de prestation } \\
\text { des soins }\end{array}$ \\
\hline 1. & Protection des enfants 0-5 ans par MIILD & Famille et communauté \\
\hline 2. & Prise en charge présomptive du paludisme chez les enfants de 0-59 mois & Famille et communauté \\
\hline 3. & Prise en charge de la diarrhée chez les enfants de 0-59 mois & Famille et communauté \\
\hline 4. & Prise en charge des IRA chez les enfants de 0-59 mois & Famille et communauté \\
\hline 5. & Dépistage et prise en charge de la malnutrition aiguë modérée & Famille et communauté \\
\hline 6. & Allaitement maternel exclusif (0-6 mois) & Famille et communauté \\
\hline 7. & Planification familiale & Centre de santé (CS) \\
\hline 8. & Consultation prénatale & CS \\
\hline 9. & Vaccinations infantiles (vaccination anti rougeoleuse) & CS \\
\hline 10. & Antibiotiques pour la pneumonie des enfants de moins de 5 ans & CS \\
\hline 11. & SONUB (Soins Obstétricaux et Néonataux d'Urgence de Base) & CS \\
\hline 12. & Accouchement normal assisté par personnel qualifié & CS \\
\hline 13. & PTME (test, conseil, AZT, sd NVP et conseil sur alimentation nourrisson) & CS/HZ \\
\hline 14. & SONUC (Soins Obstétricaux et Néonataux d'Urgence Complets) & HZ \\
\hline
\end{tabular}

Source: Tiré du Document " Paquets d'IHI par niveau de soins pour l'atteinte des OMD au Bénin », mai 2010 
L'analyse du tableau 2 montre l'ampleur des interventions au niveau communautaire et famille. Ce niveau requiert encore plus d'attention au regard du nombre important de relais communautaires qui reste à recruter pour couvrir l'ensemble des localités des zones sanitaires. Les interventions au niveau famille et communauté sont très indispensables, car elles contribuent à réduire la pression sur les centres de santé et les hôpitaux. Lorsqu'on se réfère au rôle de cette catégorie dans les conseils et les petits soins de proximité, ce gap est très important au regard de la proximité du milieu avec les pays limitrophes. Par ailleurs, On note une grande disparité dans la répartition géographique du personnel disponible dans le département, surtout la catégorie des médecins qui est plus concentrée dans les grandes villes particulièrement à Porto-Novo.

\subsubsection{Moyens de transport en urgence et matériels roulant pour augmenter l'accessibilité géographique}

Les moyens de transport en urgence sont très indispensables dans les milieux éloignés des grands centres de santé. On ne peut pas référer des malades avec des moyens inadaptés, car il leur faut de moyen de transport adapté à leur état de santé pour pouvoir se déplacer et recevoir des soins. Le tableau 3 présente le besoin et la disponibilité en moyens de transport en urgence.

Tableau 3: Disponibilité en moyens de transport en urgence

\begin{tabular}{|c|c|c|c|c|c|c|c|}
\hline \multicolumn{2}{|l|}{ Zones sanitaires } & \multicolumn{3}{|c|}{$\begin{array}{l}\text { Porto-Novo-Sèmè-Podji- } \\
\text { Aguégués (PAS) }\end{array}$} & \multicolumn{3}{|c|}{$\begin{array}{l}\text { Avrankou-Apkro-Missérété- } \\
\text { Adjarra }\end{array}$} \\
\hline & & Besoin & Disponible & Gap & Besoin & Disponible & Gap \\
\hline \multirow{3}{*}{$\begin{array}{l}\text { Moyens de transport en } \\
\text { urgence et matériels } \\
\text { roulant pour augmenter } \\
\text { l'accessibilité } \\
\text { géographique }\end{array}$} & Ambulance & 3 & 1 & 2 & 2 & 0 & 2 \\
\hline & Véhicule & 7 & 0 & 7 & 6 & 0 & 5 \\
\hline & Moto & 32 & 11 & 21 & 23 & 13 & 10 \\
\hline
\end{tabular}

Source : Cartographie des intervenants et interventions en SMNI actualisée par les ZS. Juin 2017

Le gap en matériel roulant pour le déplacement en urgence (4 ambulances), pour la supervision et le ravitaillement (12 véhicules) ainsi que pour les stratégies avancées (31 motos) paraît important en dépit de la superficie relativement moyenne des deux zones sanitaires.

\subsubsection{Disponibilité en protocoles divers de travail}

Tout programme de communication efficace vise à influencer le comportement des individus et communautés en termes de recherche de soins ou de délivrance de soins. Lorsque le travail s'effectue de manière harmonieuse, les programmes de communication aboutissent aux meilleurs résultats, susceptibles d'accroître la demande et l'utilisation de services dans 
les communautés et d'améliorer parallèlement la prestation de service. Le matériel d'Information, Education, Communication/Communication pour le changement de Comportements (IEC/CCC) est presqu'inexistant dans tout le département sanitaire de l'Ouémé (Tableau 4) ; il en est de même pour les divers protocoles de travail pour le Virus de l'Immunodéficience Humaine / Syndrome d'Immunodéficience Acquise (VIH/SIDA), pour la Prise en Charge Intégrée des Maladies de l'Enfant (PCIME), la Malnutrition, le lavage de mains, etc.

En effet, les protocoles de travail sont des documents de normes conçus pour rationaliser la prise en charge clinique et thérapeutique des patients, la sensibilisation des populations en tenant compte des bonnes pratiques en cours et des leçons apprises des expériences passées, ici et dans le monde. C'est un précieux outil de travail, le protocole de prise en charge de VIH Sida par exemple, basé sur les pratiques traditionnelles efficaces définit les normes et procédures et précise les niveaux acceptables de compétences des différents acteurs dans la prise en charge des IST et des affections du VIH. Le protocole de PCIME est un manuel qui offre les explications techniques sur lesquelles reposent les directives génériques pour la prise en charge intégrée de la maladie de l'enfance.

Tableau 4: Disponibilité en protocoles divers de travail

\begin{tabular}{|c|c|c|c|c|c|c|c|}
\hline \multicolumn{2}{|c|}{ Zones sanitaires } & \multicolumn{3}{|c|}{$\begin{array}{l}\text { Porto-Novo-Sèmè-Podji- } \\
\text { Aguégués (PAS) }\end{array}$} & \multicolumn{3}{|c|}{$\begin{array}{l}\text { Avrankou- Apkro-Missérété- } \\
\text { Adjarra }\end{array}$} \\
\hline \multirow{6}{*}{$\begin{array}{l}\text { Disponibilité } \\
\text { en protocoles } \\
\text { divers de } \\
\text { travail }\end{array}$} & & Besoin & Disponible & Gap & Besoin & Disponible & Gap \\
\hline & VIH/Sida & 32 & 0 & 32 & 26 & 0 & 26 \\
\hline & Lavage des mains & 32 & 0 & 32 & 26 & 0 & 26 \\
\hline & PEV & 32 & 0 & 32 & 26 & 0 & 26 \\
\hline & PCIME & 32 & 0 & 32 & 26 & 0 & 26 \\
\hline & Malnutrition & 32 & 0 & 32 & 26 & 0 & 26 \\
\hline
\end{tabular}

Source : Cartographie des intervenants et interventions en SMNI actualisée par les ZS. Juin 2017

L'analyse du tableau 4 renseigne sur la non disponibilité totale des protocoles de travail dans les deux zones sanitaires. Pourtant, les directives élaborées conjointement par l'OMS et l'UNICEF pour la prise en charge intégrée des maladies de l'enfant (PCIME) proposent des méthodes simples et efficaces pour prévenir et soigner les principales causes de maladies graves et de mortalité chez les jeunes enfants.

\subsubsection{Disponibilité en kits SMNI}

Dans ce département sanitaire, les kits SMNI sont quasiment inexistants dans presque toutes les zones sanitaires. Lorsqu'ils existent (comme c'est le cas pour le PEV), les réfrigérateurs sont presque tous amortis avec une durée de vie moyenne de 12 ans. 
Tableau 5: Disponibilité en kits SMNI

\begin{tabular}{llll}
\hline Zones sanitaires & & $\begin{array}{l}\text { Porto-Novo-Sèmè- } \\
\text { Podji-Aguégués (PAS) }\end{array}$ & $\begin{array}{l}\text { Avrankou- Ap-kro- } \\
\text { Missérété-Adjarra }\end{array}$ \\
\hline \multirow{2}{*}{$\begin{array}{l}\text { Disponibilité } \\
\text { en kits SMNI }\end{array}$} & $\begin{array}{l}\text { Kit pour accouchement assisté (Gants } \\
\text { stériles, Cathéter, Ocytocine) }\end{array}$ & Gap : 30 & Gap : 26 \\
& $\begin{array}{l}\text { Matériel PEV } \\
\text { Matériels soins aux Nouveaux nés et } \\
\text { petits enfants }\end{array}$ & $\begin{array}{l}\text { Aspirateur Gap : } 7 \\
\text { Gap : 5 }\end{array}$ & Aspirateur Gap : 15 \\
\hline
\end{tabular}

Source : Enquête de terrain. Juin 2017

Le tableau 5 montre un gap important en Kit pour accouchement assisté (Gants stériles, Cathéter, Ocytocine), de même que le matériel de soins aux Nouveaux - nés et petits enfants dans toutes les formations sanitaires.

\subsubsection{Disponibilité en matériels pour la gestion des déchets biomédicaux et divers}

Les problèmes posés par une mauvaise gestion des déchets biomédicaux revêtent une grande acuité. Les risques liés à une mauvaise gestion de ces déchets issus des soins de santé portent globalement sur des blessures accidentelles, des intoxications aiguës, des infections et des nuisances. Les matériels pour la gestion de ces déchets sont presqu'inexistants (Tableau 6).

Tableau 6: Disponibilité en matériels de gestion des déchets biomédicaux et divers

\begin{tabular}{llll}
\hline Zones sanitaires & \multicolumn{2}{l}{$\begin{array}{l}\text { Porto-Novo-Sèmè-Podji-Aguégués } \\
\text { (PAS) }\end{array}$} & $\begin{array}{l}\text { Avrankou- Ap-kro- } \\
\text { Missérété-Adjarra }\end{array}$ \\
\hline $\begin{array}{l}\text { Disponibilité en matériels } \\
\text { pour la gestion des } \\
\text { déchets biomédicaux et } \\
\text { divers }\end{array}$ & $\begin{array}{l}\text { Boîte de } \\
\text { sécurité }\end{array}$ & Gap : 0 & Gap : 0 \\
\hline
\end{tabular}

Source : Enquête de terrain. Juin 2017

Les impacts sur l'environnement biophysique sont d'ordre divers et concernent particulièrement la pollution esthétique, la pollution de l'air et les incommodités provoqués par les brûlages à l'air libre des déchets ainsi que par les fumées des incinérateurs.

\subsubsection{Matériels pour activités d'IEC/CCC}

Les boîtes à images sur les conseils en nutrition, pour le VIH, pour les soins aux nouveaux nés etc. sont développées pour améliorer les prestataires de soins et aider les conseillers communautaires à fournir des soins complets, aussi bien au niveau des structures de santé que dans la communauté. La boîte à images est spécialement conçue pour renforcer les efforts de counseling des bénéficiaires et l'éducation communautaire concernant leurs pratiques d'alimentation et l'hygiène. Malheureusement, ces matériels ne sont pas disponibles dans un milieu où plusieurs groupes ethniques cohabitent. 
Tableau 7: Disponibilité en matériels pour activités d'IEC/CCC

\begin{tabular}{llll}
\hline Zones sanitaires & & $\begin{array}{l}\text { Porto-Novo-Sèmè- } \\
\text { Podji-Aguégués }\end{array}$ & $\begin{array}{l}\text { Avrankou- Ap-kro- } \\
\text { Missérété-Adjarra }\end{array}$ \\
\hline $\begin{array}{l}\text { Matériels } \\
\text { pour activités } \\
\text { d'IEC/CCC }\end{array}$ & Boîte à image pour soins NNé & Gap : 816 & Gap : 1513 \\
\hline
\end{tabular}

Source : Enquête de terrain. Juin 2017

Les milieux frontaliers étant composés des personnes des différentes nationalités avec des disparités linguistiques, les boîtes à image sont des outils indispensables pour faciliter la sensibilisation.

L'augmentation de la population et sa dynamique appellent de nouveaux services et de nouveaux équipements en matière de la sécurité, de la scolarisation, de logements, mais aussi et surtout en matière de la santé. L'analyse des différentes données relatives aux infrastructures et ressources en santé montre la faiblesse de l'offre sanitaire dans la zone transfrontalière.

\section{Discussions}

De l'analyse des ressources humaines de qualité, il ressort que les grandes tendances d'écarts à combler dans les zones sanitaires sont relatives aux ressources humaines qualifiées (médecins, sages-femmes et infirmiers). Les gaps varient d'une zone sanitaire à une autre mais avec des écarts parfois très importants entre les zones sanitaires. Dans le domaine de la santé, la question des ressources humaines est centrale, en quantité aussi bien qu'en qualité. Les besoins en personnel de santé pour couvrir l'ensemble des structures sanitaires et répondre à la norme 25 personnels qualifiés pour 10000 Habitants (norme OMS) restent importants pendant que la répartition du personnel existant n'est pas équitable sur l'ensemble du territoire. Dans les 2 zones sanitaires auxquelles appartient la zone d'étude, le nombre de médecins disponibles est acceptable, mais leur répartition pose problème. En effet, pendant que la zone sanitaire de Porto-Novo-Sèmè-Podji-Aguégués (PAS) enregistre un surplus en médecin, on note un gap d'environ 20 médecins dans la zone sanitaire d'Avrankou- Apkro-Missérété-Adjarra. La couverture des deux zones sanitaires en sages-femmes et infirmiers est satisfaisante. Cependant, leur répartition dans les centres de santé doit tenir compte des particularités des espaces frontaliers. Ainsi, dans les espaces frontaliers des communes d'Adjarra et de Sèmè-Podji, le ratio est de 8 , soit environ le tiers de la norme. Les relais communautaires dont l'utilisation est d'une importance et d'une nécessité absolue dans la mise en œuvre des plans sanitaires au niveau famille et communauté sont presque absents dans toutes les localités. Toutes les formations sanitaires sont concernées de façon presque égale. Les premiers soins se donnent au niveau famille/communauté par les relais communautaires (promotion des pratiques familiales essentielles, soutien aux équipes de santé 
en stratégie avancée et mobile, prise en charge des affections courantes de l'enfant etc.). Le gap en matériel roulant pour le déplacement en urgence (4 ambulances), pour la supervision et le ravitaillement (12 véhicules) ainsi que pour les stratégies avancées (31 motos) paraît important en dépit de la superficie relativement moyenne des deux zones sanitaires. Au total, les zones frontalières au sud-est du Bénin ne sont pas suffisamment équipées en équipements sanitaires adéquat. Ces résultats viennent confirmer ceux de (Benyoussef \& al, 1973), (Cuttitta, 2008) et (Bochaton, 2015).

Les localités transfrontalières du Bénin et de ses voisins étant des régions caractérisées par une forte densité et une grande interconnexion transfrontalière de leurs populations. L'absence de services de santé adéquats dans une telle zone en expansion rapide peut affecter l'état de santé des populations. Ces résultats confirment ceux de (Abdoul, 2015) sur la stratégie de résilience territoriale dans l'espace transfrontalier Kossi/Tominian (Burkina-Mali). Abdoul (2015) est parvenu à la conclusion que les zones frontalières ont la réputation d'être des zones d'insécurité, de banditisme et de trafics en tous genres et qui sont très pauvres en infrastructures et en services sociaux de base. Cette absence de services de santé adéquats dans les zones frontalières du Bénin a été également mise en exergue par l'Agence Béninoise de Gestion Intégrée des Espaces Frontaliers (ABeGIEF) et par (ARFE, 2012) sur les possibilités de coopération transfrontalière en Afrique de l'ouest.

\section{Conclusion}

Les espaces frontaliers au sud-est du Bénin connaissent un accroissement rapide de leurs populations. Ceux des communes d'Adjarra et de Sèmè-Podji sont caractérisés par une forte densité et une grande interconnexion transfrontalière de leurs populations. La mondialisation croissante des échanges engendre une augmentation des flux internationaux de populations humaines, animales et de marchandises qui peuvent favoriser la propagation des maladies épidémiques. Les espaces frontaliers sont les plus exposés, car l'apparition d'une crise sanitaire dans ces milieux peut avoir des aboutissements sur la santé des populations des autres localités. Les dernières épidémies de maladie hémorragique à virus Ebola et celle à virus Lassa sont des exemples qui démontrent que les espaces frontaliers sont constamment exposés aux risques épidémiologiques. Dans cette optique, le système de santé national devra développer des capacités de riposte aux attaques épidémiologiques. Les efforts sont fournis dans ce sens, surtout en matière de la vaccination. Cependant, la particularité des espaces frontaliers nécessite des dispositifs particuliers en matière de la prévention, de la détection et la riposte aux urgences de santé. La situation actuelle, caractérisée par l'insuffisance en ressources humaines de qualité, le manque de moyens matériels et de cadres adéquats de travail est loin d'offrir cet idéal. L'offre de santé dans les espaces 
frontaliers au sud-est du Bénin est confrontée à l'insuffisance en ressources humaines de qualité, aux difficultés d'intervention au niveau famille et communauté au regard du nombre important de relais communautaires qui restent à former et à pourvoir en kits de relais, à la quasi inexistence des kits SMNI dans presque toutes les formations sanitaires.

Le développement des modes de réponse aux catastrophes et autres urgences fait apparaître plus que jamais la nécessité de travailler ensemble sans tenir compte des frontières. Les initiatives de coopération entre le Bénin et ses voisins dans le domaine de la surveillance intégrée des maladies et la riposte, n'est pas encore à la hauteur de cette importante interconnexion transfrontalière des populations.

$\mathrm{Au}$ regard de ces constats peu reluisants, il est nécessaire d'adapter l'offre de soins et l'organisation locale du système de santé pour tenir compte de l'ensemble des acteurs présents localement et des besoins des populations transfrontaliers.

\section{References:}

1. Abdoul, M. (2015). La coopération transfrontalière : une stratégie de résilience territoriale pour la sécurité alimentaire : cas de l'espace transfrontalier Kossi /Tominian (Burkina - Mali) GIZ/PFUA. Sahel and West Africa Club Forum 2015 (p. 7). Ouagadougou: GIZ/PFUA.

2. ARFE. (2012). Possibilités de coopération transfrontalière en Afrique de l'ouest : Une contribution au processus d'intégration régionale. Germany.

3. Benyoussef, A., \& al. (1973). Santé, migration et urbanisation. Une étude collective au Sénégal. Buletin de l'Organisation Mondiale de la Santé, pp. 517-537.

4. Bochaton, A. (2015). «Migrations sanitaires et nouveaux enjeux autour la frontière lao-thaïlandaise ». L'Espace Politique [En ligne] consulté le 08 septembre 2017. URL : http://espacepolitique.revues.org/3280.

5. Cuttitta, P. (2008). Le monde-frontière. Le contrôle de l'immigration dans l'espace globalisé. [Traduit de l'anglais par Francesco Ragazzi]. Cultures \& conflits, hiver 2007(68), pp. 61-84.

6. Denert, O., \& Hurel, H. (2000). De l'espace frontalier au territoire transfrontalier. Labyrinthe, pp. 141-148.

7. Rashid, S., Hadi, A., Afsana, K., \& Begum, S. A. (2001). Infections respiratoires aiguës dans les zones rurales du Bengladesh : compréhension culturelle, pratiques et rôles des mères et des volontaires de santé communautaires. Médecine tropicale et santé internationale, 6 (4) , pp: 249-255. 
8. Robin, N. (2009). Les enjeux régionaux des migrations ouestafricaines. Cahiers de l'Afrique de l'Ouest, pp.130-149.

9. Rodriguez, N. (2012). Contrôle des frontières. Hommes et migrations. 1296, pp. 54-63.

10. Samb, M. (2010). Migration et santé en Afrique de l'ouest : une revue de la littérature, Sénégal. 50 p.

11. Schrag, S., \& Wiener, P. (1995). Emerging infectious disease : What are the relative roles of ecology and evolution ? Trends in Ecology and Evolution (Personal Edition) 10, pp. 319-324.

12. Wanner, P., Bouchardy, C., \& Droin, N. (2000). Les habitudes de vie et comportements en matière de prévention des étrangers en Suisse. Démos bulletin d'information démographique, 2/98.

13. Wessen, A. (1974). The role of migrant studies in epidemiological research. Israel Journal of Medical Science 1:, 584.

14. Wihtol de Wenden, C. (2009). La Globalisation humaine. Presses Universitaires de France (PUF), 262 pages.

15. Wihtol de Wenden, C. (2012). Les dynamiques migratoires dans le monde. Humanitaire [En ligne], 33 | 2012, mis en ligne le 06 novembre 2012, consulté le 13 septembre 2017. URL : http://humanitaire.revues.org/1412. 